\title{
Optical generation of high frequency ultrasound using two-dimensional gold nanostructure
}

\author{
Yang $\mathrm{Hou}^{\mathrm{a})}$ and Jin-Sung Kim \\ Department of Electrical Engineering and Computer Science, University of Michigan, Ann Arbor, Michigan \\ 48109 \\ Shai Ashkenazi and Matthew O’Donnell \\ Department of Biomedical Engineering, University of Michigan, Ann Arbor, Michigan 48109 \\ L. Jay Guo \\ Department of Electrical Engineering and Computer Science, University of Michigan, Ann Arbor, Michigan \\ 48109
}

(Received 15 May 2006; accepted 10 July 2006; published online 31 August 2006)

\begin{abstract}
A two-dimensional (2D) gold nanostructure is used to optically generate high frequency ultrasound. The structure consists of 2D arrangements of gold nanoparticles, sandwiched between a transparent substrate and a $4.5 \mu \mathrm{m}$ thick polydimethylsiloxane (PDMS) layer. The acoustic signal displays significant improvements compared to a bulk black PDMS films (the current state of the art) at frequencies from 50 to $100 \mathrm{MHz}$. The high optical extinction ratio of the gold nanostructure provides a convenient method to construct an integrated transmit/receive optoacoustic array. These results show that a $2 \mathrm{D}$ gold nanostructure can be used to produce high frequency arrays for ultrasound imaging. () 2006 American Institute of Physics. [DOI: 10.1063/1.2344929]
\end{abstract}

The real-time imaging capability of ultrasound makes it an attractive modality for a minimally invasive microscope. Although the technology is mature to build piezoelectric arrays operating at less than $10 \mathrm{MHz},{ }^{1,2}$ it is extremely difficult to produce two-dimensional (2D) arrays operating above $30 \mathrm{MHz},{ }^{3,4}$ where the major problems include dicing piezoceramics to micron scale elements, electrical connections, and cross-talk between elements. Thus currently, at higher frequencies, acoustic microscopes rely on mechanical scanning of a single element transducer, which could be time consuming and ultimately limit image quality. However, in vivo three-dimensional imaging at sufficiently high frame rates requires $2 \mathrm{D}$ phased arrays operating at frequencies higher than $50 \mathrm{MHz}$, which poses a huge challenge for piezoelectric technology.

A promising alternative is an optoacoustic array relying on optical generation and detection of ultrasound. ${ }^{5-9}$ These arrays use two laser beams, one for generation and the other for detection. Their size and location define transmit and receive elements. Conventional optics and scanning systems can achieve element size and spacing on the order of several microns. Optoacoustic arrays can easily overcome some major difficulties of piezoelectric equivalents because there is no need to dice the transducing surface or make any electrical connections.

The most common mechanism of optical generation of ultrasound is the thermoelastic effect. ${ }^{10-12}$ Thermoelastic generation is easy to implement and can be very broadband. However, its major drawback has always been poor conversion efficiency when a metallic film is used as the transducer. We have previously shown that the transduction efficiency can increase by $30 \mathrm{~dB}$ using an $11 \mu \mathrm{m}$ thick spin-cast film consisting of a mixture of carbon black and polydimethylsiloxane (PDMS). ${ }^{5-8}$ This device can produce acoustic power

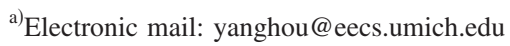

comparable to an ideal piezoelectric element at frequencies in the $100 \mathrm{MHz}$ regime.

The bandwidth of the generated ultrasound is mainly limited by the thickness of the acoustically absorbing PDMS film. The optical absorption depth of the black PDMS film defines the size of the acoustic source, which is estimated to be about $1 \mu \mathrm{m}$ from the substrate based on the shape of the acoustic radiation pattern. Thus the remaining film does not contribute to the thermoelastic effect but simply attenuates the generated ultrasound, especially high frequency components, by about $1 \mathrm{~dB} / \mu \mathrm{m} .{ }^{8}$ With carbon black as the optical absorber, the film thickness cannot be reduced to less than $11 \mu \mathrm{m}$ because the addition of carbon black significantly increases the viscosity of the mixture. This motivates us to consider other absorbing structures with reduced film thickness to further increase bandwidth.

In this letter we show a novel optoacoustic transmission structure, which consists of a 2D gold nanoparticle array sandwiched between a transparent substrate and a $4.5 \mu \mathrm{m}$ thick PDMS layer. Gold nanoparticles serve as efficient optical absorbers because surface plasmons localized around the particles strongly absorb light at a resonant wavelength that depends on the size, shape, and local dielectric environment of the gold nanoparticles. ${ }^{13,14}$ When a pulsed laser beam is focused onto the gold nanostructure layer through the glass substrate at the resonant frequency, a large fraction of incident energy is absorbed. Energy transferred over a very short time interval from the absorbing layer to PDMS rapidly heats a localized volume, in which an impulsive thermal expansion launches an acoustic pulse into the overlying sample.

To prepare the gold nanoparticle layer, a $200 \mathrm{~nm}$ thick polymer layer, with 2D arrangements of air holes spaced every $220 \mathrm{~nm}$, is fabricated on a glass substrate using nanoimprint lithography. ${ }^{15-17}$ Each hole is $200 \mathrm{~nm}$ high and $128 \times 110 \mathrm{~nm}^{2}$ in cross section. A $20 \mathrm{~nm}$ layer of gold is deposited on top of the polymer structure using an electron 


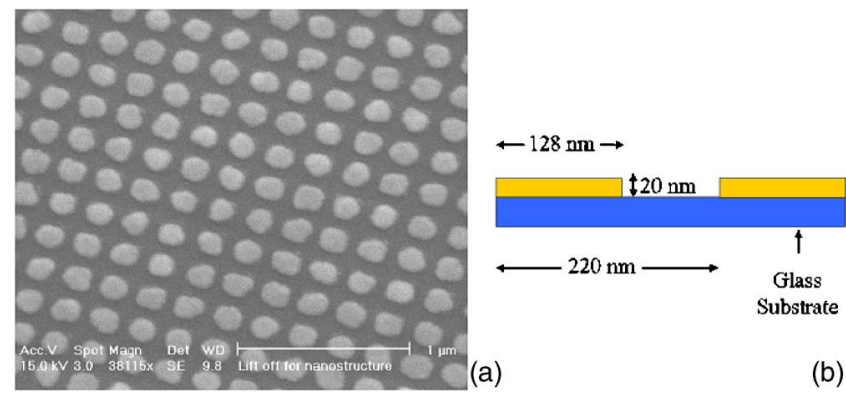

FIG. 1. (Color online) SEM picture of the top view (a) and sketch of the side view (b) of the gold nanostructure.

beam evaporator. Lift-off was then performed by soaking the sample in acetone within a low-power ultrasonic bath, removing the polymer layer as well as the overlying gold layer. A scanning electron microscope (SEM) picture of the top view of the structure is shown in Fig. 1(a), and a sketch of the side view is shown in Fig. 1(b). A mixture of PDMS and toluene, with a ratio of $2: 1$, is then spin coated into a $4.5 \mu \mathrm{m}$ thick layer on top of the gold nanoparticle layer.

Optical transmission measurements were done using a Nikon TE300 Eclipse inverted microscope (20× objective, numerical aperture $=0.44)$ with transmitted light coupled into an Ocean Optics SD2000 fiber coupled spectrometer using an achromatic lens. As illustrated in Fig. 2, transmission through the lift-off structure varies with wavelength. Maximum absorption (minimum transmission) occurs at $675 \mathrm{~nm}$, and there is a factor of 3 greater transmission at $980 \mathrm{~nm}$ compared to $675 \mathrm{~nm}$.

The experimental setup for thermoelastic generation of ultrasound is shown in Fig. 3. The gold nanostructure film is mounted on a sample holder at the water surface. The laser excitation source is a commercial high energy solid state laser with tunable wavelength (Surelite, with OPO Plus, Continuum, Inc.), which produces a 5 ns laser pulse with energy of $100 \mathrm{~mJ}$. The beam is coupled into a multimode fiber with core size of $200 \mu \mathrm{m}$ and outputs through a collimator; this reduces the pulse energy to about $100 \mu \mathrm{J}$. Before the pulse is focused onto the film, it goes through a set of neutral density filters (optical density $=3.0$ ), further reducing the energy to about $100 \mathrm{~nJ}$. It is then focused onto the gold nanostructure film through the glass slide with a $3.1 \mathrm{~mm}$ focal length aspherical lens. A $f / 1.4, \mathrm{LiNbO}_{3}$ transducer with a focal length of $4.1 \mathrm{~mm}$ and a center frequency of $80 \mathrm{MHz}$ records the acoustic signal at a distance $4.1 \mathrm{~mm}$ from the absorbing film (i.e., at the focal length of the acoustic lens).

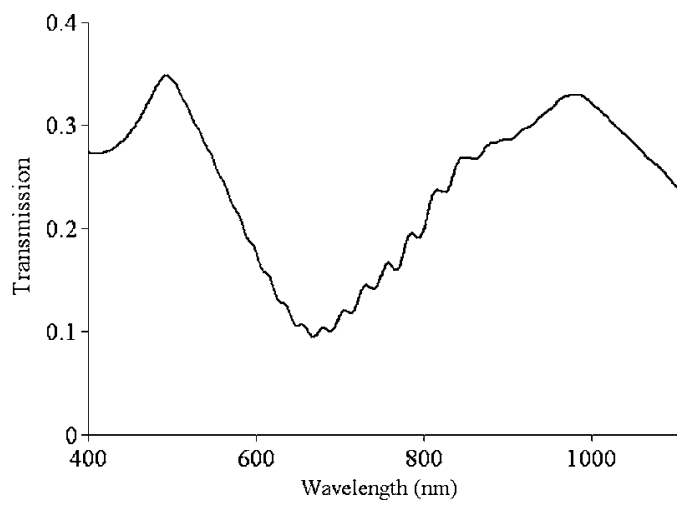

FIG. 2. Optical transmission vs wavelength.

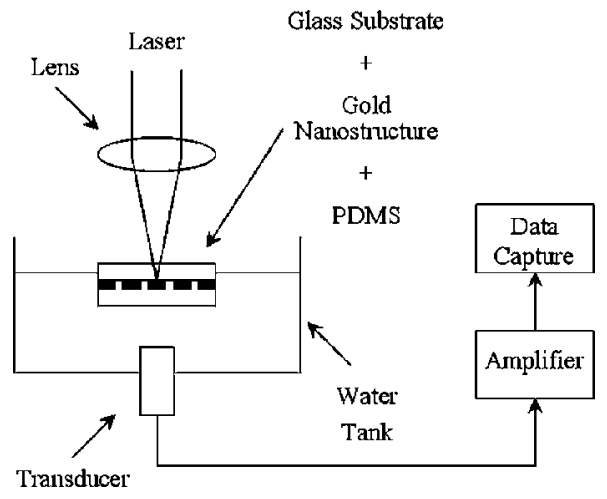

FIG. 3. Experiment setup for thermoelastic generation of ultrasound.

The detected signal is amplified by $30 \mathrm{~dB}$ before data capture.

Figure 4(a) shows the detected signal from the gold nanostructure film, averaged 1000 times, for optical excitation at $700 \mathrm{~nm}$. In comparison, the detected signal from the black PDMS film, also averaged 1000 times, is shown in Fig. 4(b). Corresponding spectra for the two films are shown in Fig. 4(c). Although the gold nanostructure film is not able to absorb $100 \%$ of the laser energy as the black PDMS film does, the acoustic signal from the gold nanostructure film is still larger than that from the black PDMS film. We attribute this to the reduced thickness of the gold nanostructure film, which means less attenuation to generated acoustic waves.

The reduced thickness has an even bigger impact on the signal spectrum. As can be observed from Fig. 3(c), the spectrum from the gold nanostructure film shows significant improvement at frequencies above $50 \mathrm{MHz}$. This also reconfirms that the higher frequency components are attenuated more in the PDMS layer overlying the acoustic source. The heat transition time from the gold nanoparticles to PDMS is about $100 \mathrm{fs}$, which is much shorter than the pulse duration. Fast heat transfer like this will generate ultrasound with higher frequency components.

Because the absorption of the gold nanostructure film depends on the incident laser wavelength and the amplitude of the acoustic signal should be a linear function of the absorbed energy, we expect the output amplitude to change with wavelength similar to the optical absorption. With our laser system, we were able to change the incident wavelength from 675 to $1000 \mathrm{~nm}$ and measure the acoustic amplitude at

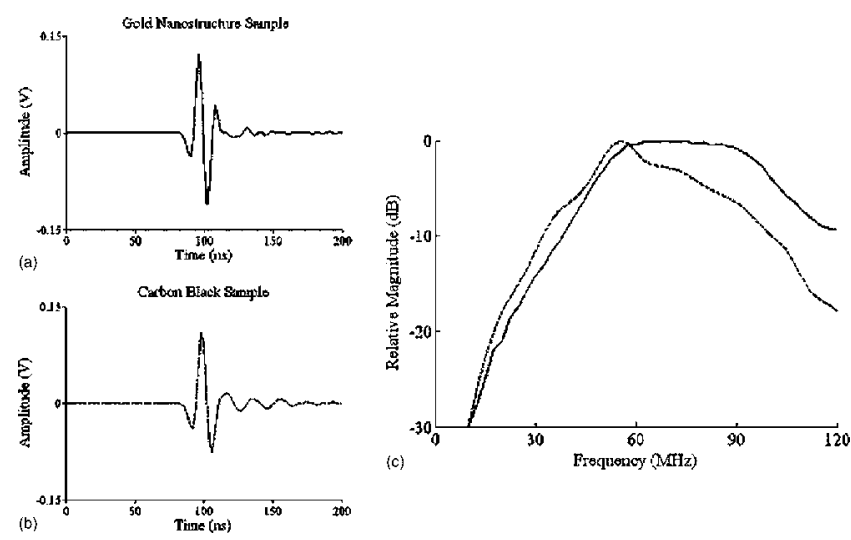

FIG. 4. Signal from (a) $4.5 \mu \mathrm{m}$ film with a single layer of gold nanoparticles and (b) $11 \mu \mathrm{m}$ black PDMS film. (c) Spectrum comparison of the gold nanostructure film (solid curve) and the black PDMS film (dashed curve). 


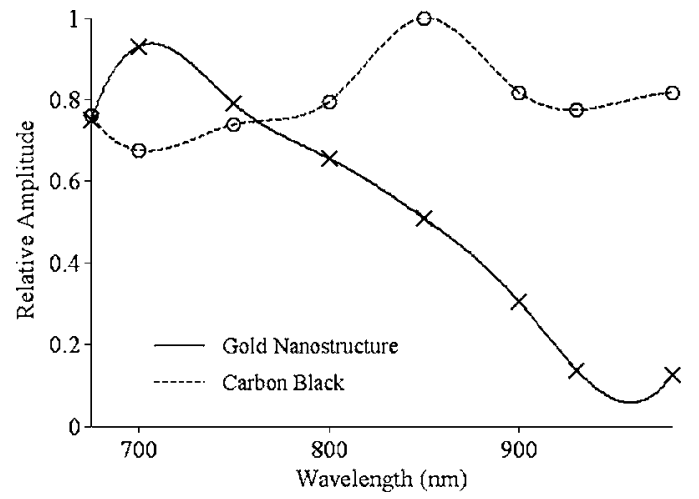

FIG. 5. Acoustic signal vs wavelength from the gold nanostructure film (dashed) and the black PDMS film (dashed).

different wavelengths. Figure 5 shows how the output changes with incident wavelength for both the gold nanostructure film and the black PDMS film. Note that the laser energy at different wavelengths is different, so we normalized the output to an incident energy of $100 \mathrm{~nJ}$. For the black PDMS film, the output amplitude does not change much with wavelength, which is expected because the film is $100 \%$ absorbing over this whole wavelength range. For the gold nanostructure film, the maximum output is located near $700 \mathrm{~nm}$, while the minimum output is near $1000 \mathrm{~nm}$. The corresponding amplitudes have a ratio of 7.5:1. The maximum and minimum locations agree fairly well with the optical transmission curve, but the extinction ratio is larger than expected. This mismatch can be corrected if optical reflection from the gold nanoparticle layer is taken into account.

The high extinction ratio of the gold nanostructure film provides a possible method to integrate it with optoacoustic detection arrays for ultrasonic imaging. Optical detection of ultrasound typically requires a separate laser beam from the one used for ultrasound generation. ${ }^{9,18}$ Ultrasound strain interacts with the optical field to modulate the optical phase; optical methods are then applied to demodulate this phase information to extract the ultrasonic signal.

We propose an optoacoustic array element containing a detection structure on top of the gold nanostructure film reported here. The detection could be a very thin polymer etalon, ${ }^{11}$ where the detection wavelength is tuned to the minimum absorption wavelength of the gold nanostructure film. Because of the high extinction ratio of the gold nanostructure film, most of the laser energy at the maximum absorption wavelength is transduced into ultrasound, whereas most of the laser energy at the minimum absorption is transmitted for ultrasound detection. An ideal element would absorb $100 \%$ of the generation beam and transmit $100 \%$ of the detection beam. A structure with optical absorption close to the ideal might be achieved by optimizing the gold nanoparticles' size, spacing, and shape.

We have previously measured the acoustic pressure from the black PDMS film, ${ }^{8,10}$ and we can estimate the acoustic pressure under current conditions based on amplitude com- parisons. When a $5 \mathrm{~ns}$ pulse with optical power of $100 \mathrm{~nJ}$ is delivered to a spot size of $25 \mu \mathrm{m}$, the acoustic pressure generated using the gold nanostructure film is about $2 \mathrm{kPa}$ at a distance of $10 \mathrm{~mm}$, representing about $1.5 \mathrm{MPa}$ at the film surface. The acoustic pressure will increase linearly with input laser energy, and the ultimate pressure possible is determined by the thermal damage threshold of the film, which reaches an optical power of $25 \mu \mathrm{J}$ delivered to a spot size of $25 \mu \mathrm{m}$. At this light level, the acoustic pressure can reach $500 \mathrm{kPa}$ at a distance of $10 \mathrm{~mm}$ and $375 \mathrm{MPa}$ at the surface, which should be sufficient for real-time ultrasound imaging.

In summary, we have demonstrated optical generation of high frequency ultrasound using a 2D gold nanoparticle array. The acoustic signal shows significant improvements over black PDMS films (the current state of the art) at frequencies above $50 \mathrm{MHz}$. We have also proposed a convenient method to integrate the gold nanostructure film with optoacoustic detection structures for ultrasound imaging arrays operating in the $100 \mathrm{MHz}$ regime.

Future work includes optimizing the pattern of gold nanoparticles to create a high extinction ratio, studying the thermal damage threshold for maximal power output, and integrating these devices with optoacoustic detection arrays for ultrasound imaging.

This work is supported in part by NIH under Grant Nos. EB003455, EB003449, and EB004933. The authors thank the Resource Center for Medical Ultrasonic Transducer Technology at the University of Southern California for supplying the high frequency transducers.

${ }^{1}$ R. A. Lemons and C. F. Quate, Appl. Phys. Lett. 24, 163 (1974).

${ }^{2}$ A. Korpel, L. W. Kessler, and P. R. Palermo, Nature (London) 323, 110 (1971).

${ }^{3}$ T. A. Ritter, T. R. Shrout, R. Tutwiler, and K. K. Shung, IEEE Trans. Ultrason. Ferroelectr. Freq. Control 49, 217 (2002).

${ }^{4}$ J. O. Fiering, P. Hultman, W. Lee, E. D. Light, and S. W. Smith, IEEE Trans. Ultrason. Ferroelectr. Freq. Control 47, 764 (2000).

${ }^{5}$ T. Buma, M. Spisar, and M. O’Donnell, Appl. Phys. Lett. 79, 548 (2001).

${ }^{6}$ T. Buma, M. Spisar, and M. O'Donnell, IEEE Trans. Ultrason. Ferroelectr. Freq. Control 50, 1065 (2003).

${ }^{7}$ T. Buma, M. Spisar, and M. O'Donnell, IEEE Trans. Ultrason. Ferroelectr. Freq. Control 50, 1161 (2003).

${ }^{8}$ Y. Hou, S. Ashkenazi, and M. O'Donnell, IEEE Trans. Ultrason. Ferroelectr. Freq. Control (submitted).

${ }^{9}$ S. Ashkenazi, Y. Hou, T. Buma, and M. O’Donnell, Appl. Phys. Lett. 86, 134102 (2005).

${ }^{10}$ R. M. White, J. Appl. Phys. 34, 3559 (1963).

${ }^{11}$ G. C. Wetsel, IEEE Trans. Ultrason. Ferroelectr. Freq. Control 33, 450 (1986).

${ }^{12}$ J. D. O'Keefe and C. H. Skeen, Appl. Phys. Lett. 21, 464 (1972).

${ }^{13}$ S. Link and M. A. El-Sayed, Int. Rev. Phys. Chem. 19, 409 (2000).

${ }^{14}$ W. Gotschy, K. Vonmetz, A. Leitner, and F. R. Aussenegg, Opt. Lett. 21, 1099 (1996).

${ }^{15}$ J. S. Kim, K. D. Lee, S. W. Ahn, S. H. Kim, J. D. Park, S. E. Lee, and S. S. Yoon, J. Korean Phys. Soc. 45, 890 (2004).

${ }^{16}$ S. W. Ahn, K. D. Lee, J. S. Kim, S. H. Kim, S. H. Lee, J. D. Park, and P. W. Yoon, Microelectron. Eng. 78, 314 (2005).

${ }^{17}$ S. W. Ahn, K. D. Lee, J. S. Kim, S. H. Kim, S. H. Lee, J. D. Park, and P. W. Yoon, Nanotechnology 16, 1874 (2005).

${ }^{18}$ J. P. Monchalin, Appl. Phys. Lett. 45, 14 (1985). 